\section{Therapieunterbrechungen beim Hodgkin- Lymphom zumindest teilweise ungefährlich}

\author{
Chemotherapien zur Behandlung des fortgeschrittenen Hodgkin-Lymphoms \\ sind wirksam - aber auch sehr toxisch. Oft muss die Behandlung deshalb un- \\ terbrochen werden. Was bedeutet das für den Erfolg der Therapie?
}

$\mathrm{D}$ ie beiden wichtigsten Kombinationsregimes zur Behandlung des Hodgkin-Lymphoms sind ABVD (Adriamycin, Bleomycin, Vinblastin und Dacarbazin) und BEACOPP (Bleomycin, Etoposid, Adriamycin, Cyclophosphamid, Vincristin, Procarbazin und Prednison). Beide enthalten das lungentoxische Bleomycin sowie ein neurotoxisches Vincaalkaloid wie Vinblastin oder Vincristin. Heinz Haverkamp und Kollegen von der Deutschen Hodgkin-Studiengruppe untersuchten anhand der HD12- und der HD15-Studie retrospektiv, inwieweit sich Dosisreduktionen von Bleomycin und Vincristin im BEACOPP-Protokoll (8 Zyklen) auf den Erfolg der Therapie auswirken.
Bei 17,6\% der 3.309 Patienten wurde die Behandlung mit Bleomycin unterbrochen und bei 32,6\% die mit Vincristin. 157 Patienten $(4,7 \%)$ erhielten $\leq 4$ Zyklen Bleomycin und $218(6,6 \%) \leq 3$ Zyklen Vincristin.

Nach einem medianen Follow-up von 59 bzw. 67 Monaten zeigten sich hinsichtlich des progressionsfreien Überlebens (PFS) bzw. Gesamtüberlebens (OS) keine signifikanten Unterschiede zwischen den Patienten, die $\leq 4$ oder $>4 \mathrm{Zy}$ klen Bleomycin erhalten hatten. Das PFS zeigte nach 5 Jahren eine Differenz von 1,7 Prozentpunkten und das OS von 1,5

Auch bei Patienten, die $\leq 3$ oder $>3$ Zyklen Vincristin erhalten hatten, ergaProzentpunkten. ben sich keine signifikanten Unterschiede: Sie lagen nach 5 Jahren bei -1,3 Prozentpunkten für das PFS und bei $-0,1$ Prozentpunkten für das OS.

Fazit: Eine Unterbrechung von Bleomycin oder Vincristin aufgrund medikamentenspezifischer Nebenwirkungen beeinflusst die Wirksamkeit der Therapie nicht. Die Gründe dafür sind bislang unklar. Es ist möglich, dass Bleomycin und Vincristin bei jenen Patienten, die besonders unter Nebenwirkungen durch diese Chemotherapeutika leiden, auch besonders wirksam sind und deshalb weniger Zyklen ausreichen; möglicherweise haben Bleomycin und Vincristin aber auch gar keine so große Bedeutung für den Erfolg der Therapie. Dies sollte im Rahmen weiterer Studien untersucht werden.

Christina Berndt

Haverkamp H et al. Impact of Bleomycin and Vincristine Dose Reductions in Patients With Advanced Hodgkin Lymphoma Treated With BEACOPP: An Analysis of the German Hodgkin Study Group HD12 and HD15 Trials. J Clin Oncol. 2015;33(22):2430-6.

\title{
Alternatives Kombinationsregime nach gescheiterter CLL-Ersttherapie
}

Entwickeln Patienten mit einer chronischen lymphatischen Leukämie (CLL) ein Rezidiv oder sprechen sie auf die Erstlinientherapie nicht mehr an, bieten hochdosierte Kortikosteroide eine wichtige Alternative für die Weiterbehandlung. Die Kombination mit einem Anti-CD20-Antikörper kann das Ansprechen und Gesamtüberleben verbessern.

$\mathrm{H}$ ochdosiertes Methylprednisolon (HDMP) oder Dexamethason in Kombination mit Rituximab zeigen Aktivität bei der rezidivierten bzw. therapierefraktären (RR) CLL. Häufig wird dies jedoch mit schweren Infektionen und nur kurzen progressionsfreien Krankheitsintervallen erkauft. Dexamethason scheint nebenwirkungsärmer als HDMP, wirkt zudem synergistisch, direkt apoptotisch sowie antiproliferativ auf Lymphomzellen und erhöht die Sensitivität für Rituximab.

Der humane monoklonale IgG1-Antikörper Ofatumumab bindet an ein anderes Epitop auf dem CD20-Protein als Rituximab und erreicht eine höhere Ober- flächendichte, eine effizientere Komplement-abhängige Zytotoxizität und höhere Antikörper-abhängige zellvermittelte Zytotoxizität auch bei geringer CD20-Expression. Vor diesem Hintergrund entwarfen Michael Doubek und Kollegen eine multizentrische, nicht randomisierte Phase-II-Studie zur Evaluierung der Wirkung einer Ofatumumab-Dexamethason-Kombination (O-Dex) bei Patienten mit einer RR-CLL. Sekundäre Endpunkte waren Therapiesicherheit und weitere Ansprechparameter. 33 CLL-Patienten durchliefen das O-Dex-Regime in geplanten 6 Zyklen.

_Zyklus 1: $300 \mathrm{mg}$ Ofatumumab i.v. an

Tag 1, 2.000 mg an Tag 8, 15 und 22,
-Zyklen 2-6: $1.000 \mathrm{mg}$ Ofatumumab an

Tag 1, 8, 15 und 22.

Daneben erhielten die Patienten in allen 6 Zyklen oral $40 \mathrm{mg}$ Dexamethason an den Tagen 1-4 und 15-18. 67\% der Patienten erreichten ein Ansprechen, 15\% eine komplette Remission. Das mediane progressionsfreie Überleben betrug 10, das mediane Gesamtüberleben $34 \mathrm{Mo}$ nate. $33 \%$ der Patienten entwickelten Infektionen vom Grad 3-5.

Fazit: Ofatumumab plus Dexamethason führt bei Patienten mit RR-CLL zu relativ hohen Gesamt- und Komplettremissionsraten, und dies bei einem günstigeren progressionsfreien und Gesamtüberleben als publizierte Rituximab/Dexamethason-Regimes. Wolfgang Zimmermann

Doubek $\mathrm{M}$ et al. Ofatumumab added to dexamethasone in patients with relapsed or refractory chronic lymphocytic leukemia: Results from a phase II study. Am J Hematol. 2015; 90(5):417-21. 